\section{ORIGINAL RESEARCH}

\author{
L. Pierot \\ T. Liebig \\ V. Sychra \\ K. Kadziolka \\ F. Dorn \\ C. Strasilla \\ C. Kabbasch \\ J. Klisch
}

\title{
Intrasaccular Flow-Disruption Treatment of Intracranial Aneurysms: Preliminary Results of a Multicenter Clinical Study
}

\begin{abstract}
BACKGROUND AND PURPOSE: The endovascular treatment of intracranial aneurysms with unfavorable anatomy (large aneurysms, wide-neck) is frequently challenging and is also associated with a high incidence of significant recurrences. The WEB, an intrasaccular flow disrupter, was designed for use in this type of aneurysm. We report our early experience with this device in this multicenter study.
\end{abstract}

MATERIALS AND METHODS: Twenty patients with 21 aneurysms were treated by using the WEB in 3 European centers. The ability to successfully deploy the WEB, immediate posttreatment angiographic results, adverse events, clinical outcome, and angiographic follow-up results were recorded.

RESULTS: Aneurysm location was the ICA (4/21, 19.1\%), MCA (8/21, 38.1\%), AcomA (5/21, 23.8\%), and $B A(4 / 21,19.1 \%)$. No treatment failures were reported. Treatment was performed exclusively with the WEB in 16/21 (76.2\%) patients. Additional treatment (coiling and/or stent placement) was used in $5 / 21(23.8 \%)$ patients. One patient (4.8\%) experienced transient clinical worsening (mRS 1 at 1 month, mRS 0 at 3 months) related to a thromboembolic event. Inadvertent detachment of the WEB was observed, and the WEB was retrieved in 1 patient, without adverse effects. In the short-term follow-up (2-8 months), adequate occlusion (total occlusion or neck remnant) was observed in $80.0 \%$ of aneurysms.

CONCLUSIONS: Intrasaccular flow disruption is a new endovascular approach for aneurysm treatment. In our preliminary experience, this treatment was feasible and mostly used in bifurcation aneurysms (MCA, BA, ICA) with unfavorable anatomy. Further studies are needed to precisely evaluate the indications, safety, and efficacy of this new technique.

ABBREVIATIONS: AcomA = anterior communicating artery; $\mathrm{BA}=$ basilar artery; $\mid \mathrm{A}=$ intra-arterial; $\mathrm{mRS}=$ modified Rankin Scale; $\mathrm{WEB}=$ Woven EndoBridge Cerebral Aneurysm Embolization Device

A neurysm coiling now has an important place in the management of both ruptured and unruptured aneurysms. ${ }^{1-3}$ However, it has 2 important shortcomings: Coiling is not applicable to all intracranial aneurysms and is associated with a relatively high percentage of recanalization (approximately $20 \%) .{ }^{4}$ Aneurysms with a complex anatomy (fusiform, wideneck, and large and giant aneurysms) are, in some cases, untreatable or difficult to treat with standard coiling.

Wide-neck aneurysms have been, since the beginning of coiling, challenging for the endovascular approach. The balloon remodeling technique, stent-assisted coiling, and use of flow diverters have all increased the number of aneurysms that may be treated by using an endovascular technique, especially in aneurysms having a sidewall (lateral) geometry. ${ }^{5-9}$ The treatment of wide-neck bifurcation aneurysms remains challenging despite the use of complex techniques like the double-

\section{Received March 23, 2012; accepted after revision April 30.}

From the Department of Radiology (L.P., K.K.), Maison Blanche Hospital, University of Reims, France; Department of Neuroradiology (T.L., F.D., C.K.), Universitätsklinikum Köln, Cologne, Germany; and Department of Diagnostic and Interventional Radiology and Neuroradiology (V.S., C.S., J.K.), Helios General Hospital, Erfurt, Germany.

Please address correspondence to Laurent Pierot, MD, PhD, Department of Radiology, Maison Blanche Hospital, 45 Rue Cognacq Jay, 51092 Reims, France; e-mail: Ipierot@gmail.com

Indicates open access to non-subscribers at www.ajnr.org

Indicates article with supplemental on-line tables.

http://dx.doi.org/10.3174/ajnr.A3191 balloon remodeling technique or Y-stent placement. These methods are, however, frequently technically difficult, and their use remains relatively limited. ${ }^{7,10}$ The use of intraluminal flow diverters does not, with few exceptions, address aneurysms of this type. Clipping can be used in such situations, especially for MCA aneurysms.

The WEB (Sequent Medical, Aliso Viejo, California), an intrasaccular flow-disruption device, was initially developed to create a modification of blood flow at the level of the aneurysm ostium (neck). In this report, we present the initial experiences from a multicenter series of aneurysms treated with the WEB device.

\section{Materials and Methods}

The study was approved by the institutional review board of Reims University Hospital.

In the 3 participating centers, indications for treatment and the technique (surgery or endovascular treatment) were decided on a case-by-case basis by a local multidisciplinary team including neurosurgeons, neuroanesthesiologists, neurologists, and neuroradiologists. No inclusion or exclusion criteria were precisely defined. The selection of aneurysms treated with the WEB device was performed autonomously in each center by the interventional neuroradiologists according to aneurysm characteristics (aneurysm status, location and size, and neck size).

Informed consent was obtained from all patients. Risks, benefits, alternatives, and potential complications involved with the WEB embolization procedure were discussed. 

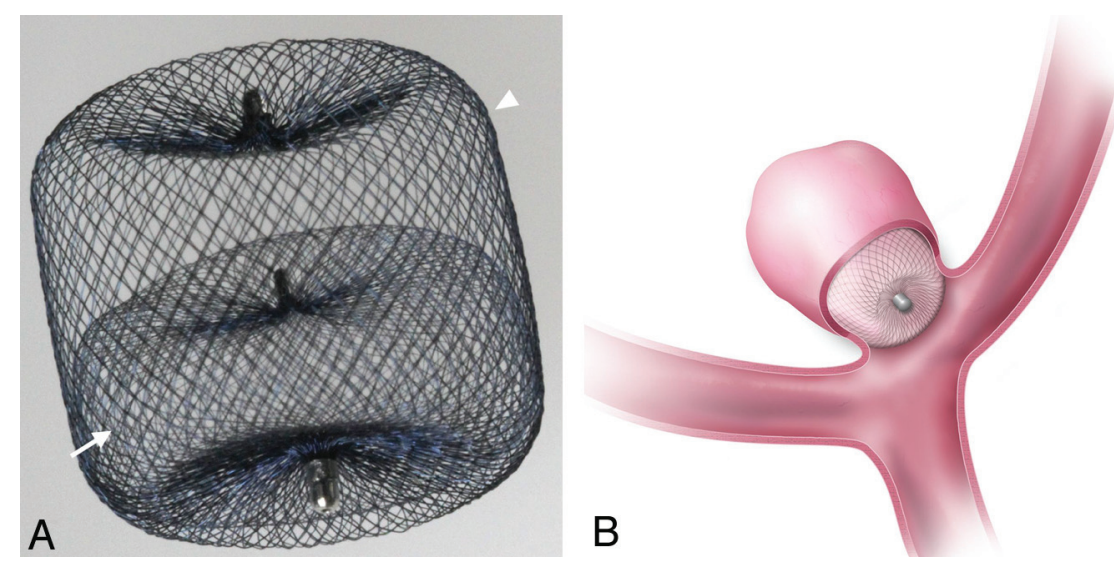

Fig 1. The WEB device. A, Proximal (arrow) and distal (arrowhead) compartments. B, Drawing shows the device in place in the aneurysm with a smooth coverage of the neck. (Figure provided with permission from Sequent Medical, Aliso Viejo, California.)

The WEB is a self-expanding, oblate, braided nitinol mesh (Fig 1). The device is composed of an inner and outer braid held together by proximal, middle, and distal radiopaque markers, creating 2 compartments: 1 distal and 1 proximal. Depending on the device diameter, the inner and outer braids are 108 wires or 144 wires. Therefore, blood flow into a WEB-embolized aneurysm initially encounters 2 layers of wires comprising 216 or 288 wires, with the largest interwire distance ranging from 106 to $181 \mu \mathrm{m}$, respectively, depending on the device size. The WEB implant is deployed-or retrieved before detachment-in a manner similar to that in endovascular coil systems, through microcatheters with an internal diameter $\geq 0.027$ inch. For devices with a diameter of $\leq 7 \mathrm{~mm}$, microcatheters with an internal diameter of 0.027 inch are used; and for devices with a diameter $>7$ $\mathrm{mm}$, microcatheters with an internal diameter 0.032 inch are used. The detachment system is electrothermal and instantaneous. The WEB received Conformité Européenne (European Conformity) Mark (CE Mark) certification for unruptured and ruptured aneurysms.

The treatment of aneurysms with the WEB was performed by using techniques similar to those used in the treatment of aneurysms with coils (eg, general anesthesia, intraoperative treatment with intravenous heparin, single or double femoral approach). In unruptured aneurysms, aspirin was usually given during the procedure and in the days after, as in standard coiling treatment. In situations in which the treating physician thought that the use of a stent might be an alternative to WEB treatment in case of failure, double antiplatelet therapy was given before and during the procedure.

An accurate evaluation of aneurysm anatomy was performed by the treating physician before aneurysm treatment by using MRA, 2D, and 3D-DSA. The morphology of the aneurysm and aneurysm transverse diameter, height, and neck size were carefully analyzed to determine whether treatment with the WEB was indicated and for appropriate device sizing.

Bench and preclinical testing showed that the WEB is best sized by using a $10 \%$ increase of the average width of the aneurysm to select the WEB width. For a given WEB width, several WEB heights are available, enabling the physician to conform the WEB to the aneurysm and seal the ostium. To facilitate the sizing process, Sequent Medical created a sizing application that predicts a WEB size on the basis of aneurysm measurements.

After the positioning of a guiding catheter into the ICA or vertebral artery, we catheterized the aneurysm by using a Rebar 27 (Covi- dien/ev3, Irvine, California), Marksman 27 (Covidien/ev3), DAC 038 (Concentric Medical, Mountain View, California), or Penumbra 41 (Penumbra, Alameda, California). The microcatheter was chosen according to the WEB size. The WEB device chosen according to aneurysm measurements was then positioned in the aneurysmal sac. A control angiogram was obtained to check the position of the device in the aneurysm and to evaluate flow stagnation inside the aneurysm. The device was correctly positioned when the 3 markers were in a line grossly perpendicular to the neck, with the proximal marker in the neck. If the position was not satisfactory, the device was resheathed and repositioned. If the size was not appropriate, the device was resheathed and removed, and another device was deployed into the aneurysm. When the right-sized device was correctly positioned, a final DSA run was performed. Treatment with ancillary devices (balloon, coils, and stent) was allowed if deemed necessary by the treating physician to improve the quality of initial aneurysm occlusion or to preserve the patency of the parent vessel.

Follow-up of patients was conducted according to the usual clinical practice of each center.

All patients treated with the WEB device in the 3 participating centers were included in the study. Data were retrospectively collected. For each patient and aneurysm, we collected the following data: age, sex, aneurysm status (ruptured/unruptured), aneurysm location and size (transverse diameter and height), neck size, modalities of treatment (perioperative medications, associated treatment), intraoperative complications (aneurysm rupture, thromboembolic events, device problems), and postoperative complications (thromboembolic complications, delayed rupture).

The quality of aneurysm occlusion was assessed by the treating physician by DSA at the end of the procedure, by MRA $24-48$ hours after the treatment, and by DSA in the short-term follow-up.

Population, feasibility of the treatment, rate of intraprocedural and postprocedural complications, morbidity and mortality, and immediate and follow-up angiographic outcome were evaluated.

At the end of the treatment and at 24-48 hours, residual flow inside the aneurysm and the device was evaluated by using a 4 -grade scale: $0=$ no residual flow inside the aneurysm and the device, $1=$ residual flow inside the proximal compartment, 2 = residual flow in the distal compartment, and $3=$ residual flow between the device and the aneurysm wall or at the dome. At short-term follow-up (months), the quality of aneurysm occlusion was evaluated by using the 3 -grade 


\begin{tabular}{|c|c|c|c|c|c|c|c|c|}
\hline \multicolumn{9}{|c|}{ Patient and aneurysm characteristics } \\
\hline No. & $\begin{array}{l}\text { Sex, Age } \\
\text { (yr) }\end{array}$ & $\begin{array}{l}\text { Aneurysm } \\
\text { Status }\end{array}$ & $\begin{array}{l}\text { Aneurysm } \\
\text { Location }\end{array}$ & $\begin{array}{l}\text { Transverse Diameter } \\
(\mathrm{mm})\end{array}$ & $\begin{array}{l}\text { Height } \\
(\mathrm{mm})\end{array}$ & $\begin{array}{l}\text { Neck Size } \\
(\mathrm{mm})\end{array}$ & $\begin{array}{l}\mathrm{D} / \mathrm{N} \\
\text { Ratio }\end{array}$ & $\begin{array}{c}\text { Previous } \\
\text { Treatment }\end{array}$ \\
\hline 1 & $F, 51$ & UnR & BA & 7.4 & 8.4 & 4.6 & $1: 6$ & No \\
\hline 2 & $F, 56$ & UnR & ICA P & 6.0 & 5.6 & 4.4 & $1: 4$ & No \\
\hline 3 & $F, 66$ & UnR & AcomA & 4.8 & 6.0 & 3.1 & $1: 6$ & No \\
\hline 4 & $F, 62$ & UnR & MCA L & 4.0 & 4.0 & 4.2 & $1: 0$ & No \\
\hline 5 & $M, 55$ & UnR & MCA L & 4.1 & 4.3 & 2.0 & $2: 1$ & No \\
\hline 6 & $F, 66$ & UnR & MCA L & 5.3 & 5.4 & 2.6 & $2: 0$ & Coils \\
\hline 7 & $F, 45$ & UnR & MCA L & 4.6 & 7.0 & 4.1 & $1: 1$ & No \\
\hline 8 & $F, 49$ & UnR & BA & 5.5 & 4.6 & 2.1 & $2: 6$ & No \\
\hline 9 & $F, 60$ & UnR & BA & 5.5 & 5.9 & 2.5 & $2: 2$ & No \\
\hline 10 & M, 57 & UnR & AcomA & 6.4 & 4.0 & 5.4 & $1: 2$ & No \\
\hline 11 & $\mathrm{~F}, 50$ & UnR & ICA B & 5.3 & 4.5 & 3.0 & $1: 8$ & No \\
\hline \multirow[t]{2}{*}{12} & $F, 75$ & UnR & MCA R & 6.8 & 7.5 & 4.0 & $1: 7$ & No \\
\hline & & UnR & MCA L & 5.5 & 5.0 & 5.5 & $1: 0$ & No \\
\hline 13 & $M, 57$ & UnR & AcomA & 5.2 & 4.8 & 4.8 & $1: 1$ & No \\
\hline 14 & $F, 65$ & UnR & ICA P & 8.4 & 9.3 & 4.8 & $1: 8$ & No \\
\hline 15 & $F, 55$ & UnR & MCA R & 4.9 & 4.9 & 5.5 & $0: 9$ & No \\
\hline 16 & $F, 35$ & UnR & MCA L & 7.3 & 7.6 & 5.7 & $1: 3$ & No \\
\hline 17 & $F, 71$ & UnR & BA & 6.1 & 7.9 & 3.0 & $2: 0$ & No \\
\hline 18 & $F, 72$ & $\mathrm{R}$ & AcomA & 5.2 & 4.9 & 4.0 & $1: 3$ & No \\
\hline 19 & $F, 56$ & UnR & ICA B & $25(15)$ & 23 & 5.0 & $3: 0$ & No \\
\hline 20 & $M, 72$ & UnR & AcomA & $35(12)$ & 26 & 4.0 & $3: 0$ & No \\
\hline
\end{tabular}

Note:-D/N indicates dome/neck; $R$, ruptured; UnR, unruptured; B, bifurcation; $P$, paraophthalmic; $L$, left; $R$, right.

aneurysm occlusion scale (total occlusion, neck remnant, aneurysm remnant). Evaluation was conducted by the operator.

\section{Results}

From October 2010 to January 2012, twenty patients (16 women and 4 men) aged 35-75 years (mean, $58.75 \pm 10.15$ years) were treated with the WEB device in 3 centers (respectively, 10, 6, and 4 patients in each center). One patient had 2 MCA aneurysms. All except 1 aneurysm were unruptured. The ruptured aneurysm was treated 1 day after rupture.

Aneurysm location, size, neck dimension, and dome-toneck ratio are shown in the Table. Two aneurysms (patients 19 and 20) were giant, partially thrombosed aneurysms located at the ICA bifurcation and AcomA, measuring, respectively, 25 and $35 \mathrm{~mm}$ with a lumen measuring, respectively, 15 and 12 $\mathrm{mm}$. Neck size was $<4 \mathrm{~mm}$ in 7 aneurysms (33.3\%) and $\geq 4$ $\mathrm{mm}$ in 14 aneurysms (66.7\%). Dome-to-neck ratio (transverse diameter/neck) was $<2.0$ in 14 aneurysms $(66.7 \%)$ and $\geq 2.0$ in 7 aneurysms (33.4\%).

No treatment failure was encountered in this series. Deployment of the WEB device was achieved in all cases. Complete repositioning of the device was not necessary in our series. In some cases, the position of the device was slightly modified inside the aneurysm by pulling or pushing on the microcatheter, the pusher, or both.

Treatment was performed exclusively with the WEB device in 16/21 aneurysms (76.2\%) (On-line Table 1 and Figs 2 and $3)$. Unexpected additional treatment was performed in $3 / 21$ aneurysms (14.3\%), including coiling in 2 aneurysms and stent placement in 1 aneurysm (Fig 4). In 1 AcomA aneurysm, the device was successfully deployed, and the control DSA just before WEB detachment showed a total aneurysm occlusion. The detachment of the device was accompanied by a small displacement of the device with the appearance of small residual flow inside the aneurysm, which was treated with coils. In 1 previously coiled MCA, the size of the aneurysm remnant was very difficult to evaluate and the size of the device used was too small; 2 additional coils were deposited after deployment of the WEB. In these 2 aneurysms, additional coiling was easily performed; a regular microcatheter was inserted in the aneurysms in both cases between the WEB and the aneurysm wall, and coils were easily deployed. In 1 compassionate-use treatment of a ruptured aneurysm, thrombus formation was observed in the anterior cerebral artery. A Solitaire (Covidien/ ev3) stent was used after IA administration of tirofiban to prevent further thrombosis in the parent vessel. The stent was deployed in the usual way.

In addition, for 2 giant partially thrombosed aneurysms (2/21: $9.5 \%)$, adjunctive therapies were planned in advance of the procedure. Coiling was performed in 1 aneurysm, and stent placement and coiling were performed in 1 aneurysm. Coiling was performed before placement of the WEB at the neck, and the stent was placed in a standard way at the end of the procedure.

At the end of the procedure, no residual flow was seen in the device and the aneurysm in 12/21 aneurysms (57.1\%) (On-line Table 2). Residual flow in the proximal compartment was observed in $7 / 21$ cases (33.3\%) (Fig 3). Residual flow was seen in the proximal and distal compartments in 2 aneurysms $(9.5 \%)$ (Fig 2).

Follow-up MRA was performed 24-48 hours after the WEB treatment. Because the device was made with nitinol (and platinum for the markers), no significant artifacts were observed in any cases. At 24-48 hours, no residual flow was seen in the device and the aneurysm in 15/21 aneurysms $(71.4 \%)$. Residual flow in the proximal compartment was observed in $5 / 21$ cases $(23.8 \%)$. Residual flow was seen in the proximal and distal compartments in 1 aneurysm $(4.8 \%)$.

In 2 patients, thrombus was observed intraoperatively, but vessel reopening was achieved without clinical worsening (On-line Table 1). In patient 12, during the treatment of a left MCA aneurysm, before insertion of the WEB, thrombus was 

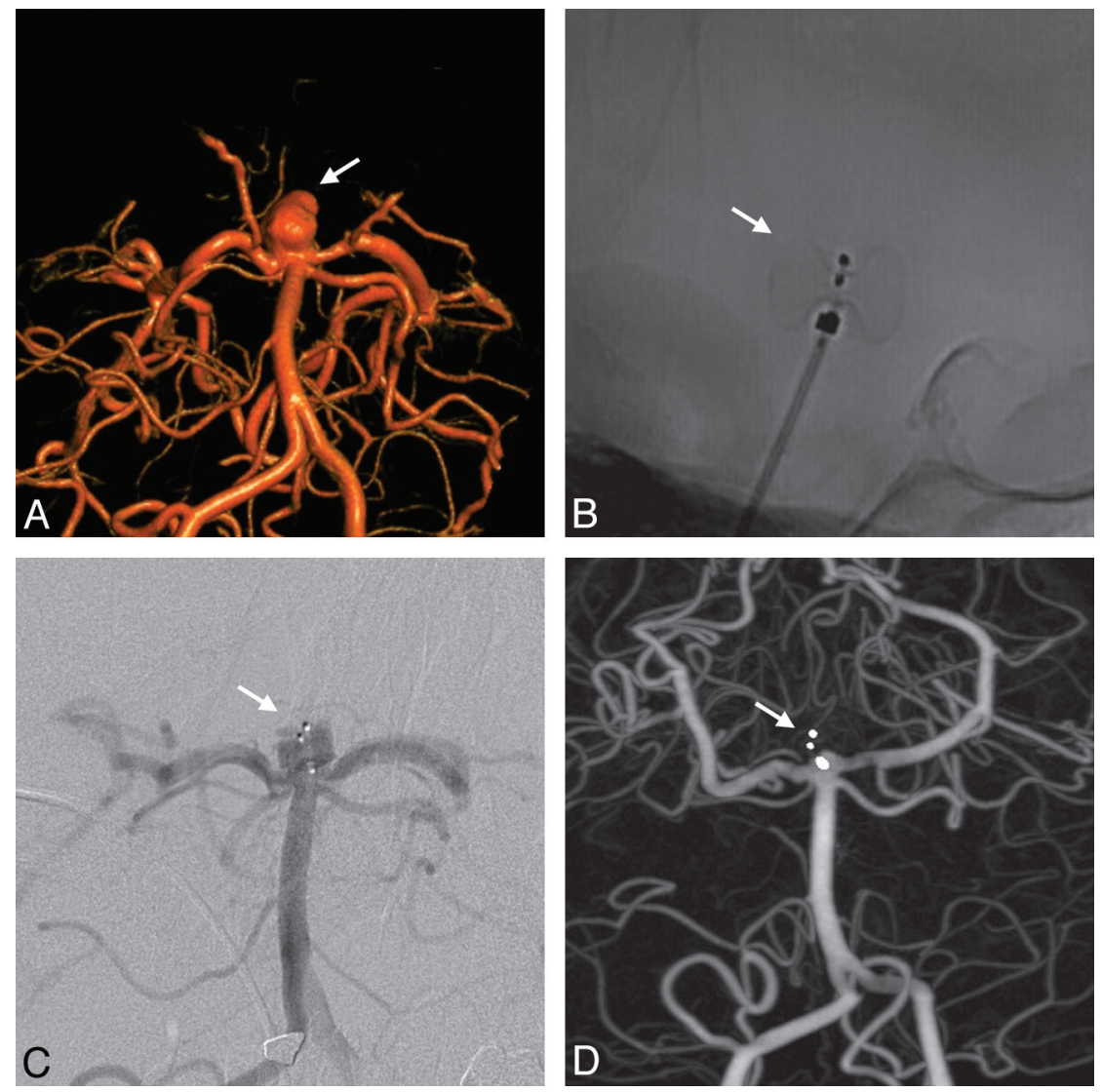

Fig 2. Unruptured basilar artery aneurysm (patient 1). A, 3D angiography shows the aneurysm (arrow). B, Lateral view after the deployment of the device (arrow). $C$, Angiography performed 30 minutes after the deployment of the device shows that the proximal and distal compartments are still filled by the contrast media (arrow). $D$, Three-month follow-up $3 \mathrm{D}$ angiography shows that the aneurysm is occluded with a very small remnant at the neck (arrow).

observed in the distal left anterior cerebral territory. The patient was treated with IA recombinant tissue plasminogen activator with complete reopening of the anterior vessel. Subsequently, the patient was successfully treated with the WEB, with no clinical sequelae. Patient 18 had a compassionate-use treatment of a ruptured aneurysm (Hunt and Hess 4). In this patient, thrombus formation was observed at the aneurysm neck during the procedure and was treated by IA tirofiban and a Solitaire stent (see above).

No intraoperative rupture occurred $(0.0 \%)$. A single postprocedural thromboembolic complication occurred in this series (4.8\%): In patient 6 , no thromboembolic complication was observed during treatment, but postoperatively, the patient was hemiparetic. Diffusion MR imaging showed multiple small ischemic lesions in the left MCA territory, but no occlusion of the MCA branches was depicted by MRA. The patient was treated with heparin and aspirin, and partial rapid clinical improvement was observed. The patient was discharged 2 days after treatment, with a modified Rankin Scale score of 1 at 1 month and 0 at 3 months.

Inadvertent device detachment occurred in 1 case (patient 1); the device was easily retrieved by using Alligator (Covidien/ ev3) and Snare (Covidien/ev3) devices, with no adverse effects.

Finally, no treatment-related mortality was observed $(0.0 \%)$. Treatment morbidity was encountered in 1 patient $(4.8 \%)$ presenting with hemiparesis after the procedure, with rapid clinical improvement (mRS at 1 month, and mRS 0 at 3 months) and no delay in hospital discharge, as described above (patient 6).

Short-term aneurysm occlusion was evaluated by DSA in $15 / 21$ aneurysms (71.4\%), 3-12 months after the treatment (On-line Table 2). Total occlusion was observed in 7/15 aneurysms (46.7\%) (Fig 3); neck remnant, in $5 / 15$ aneurysms (33.3\%) (Fig 2); and aneurysm remnant, in 3/15 aneurysms (20.0\%) (1 was aneurysm regrowth after initial complete occlusion). No correlation was found between early MRA and late DSA findings, but the number of patients was small.

During the follow-up period, retreatment was performed in 2 aneurysms (patients 8 and 14, 9.5\%). In patient 8 , a BA aneurysm was initially completely occluded and recanalization was observed at 6-month follow-up. It was retreated with 2 stents (Y-stent placement) and coils, with a good clinical and anatomic result. In patient 14, the ICA aneurysm was initially partially occluded and was retreated at 6 months by using an intravascular flow diverter (Pipeline Embolization Device; Chestnut Medical Technologies, Menlo Park, California) with a good anatomic results and no complications.

\section{Discussion}

Intrasaccular flow disruption is a new endovascular approach for aneurysm treatment, aiming at disrupting the intra-aneurysmal flow and creating intra-aneurysmal thrombosis by using a device to close the aneurysm neck from inside the aneurysm. The goal of this first preliminary series was to evaluate 

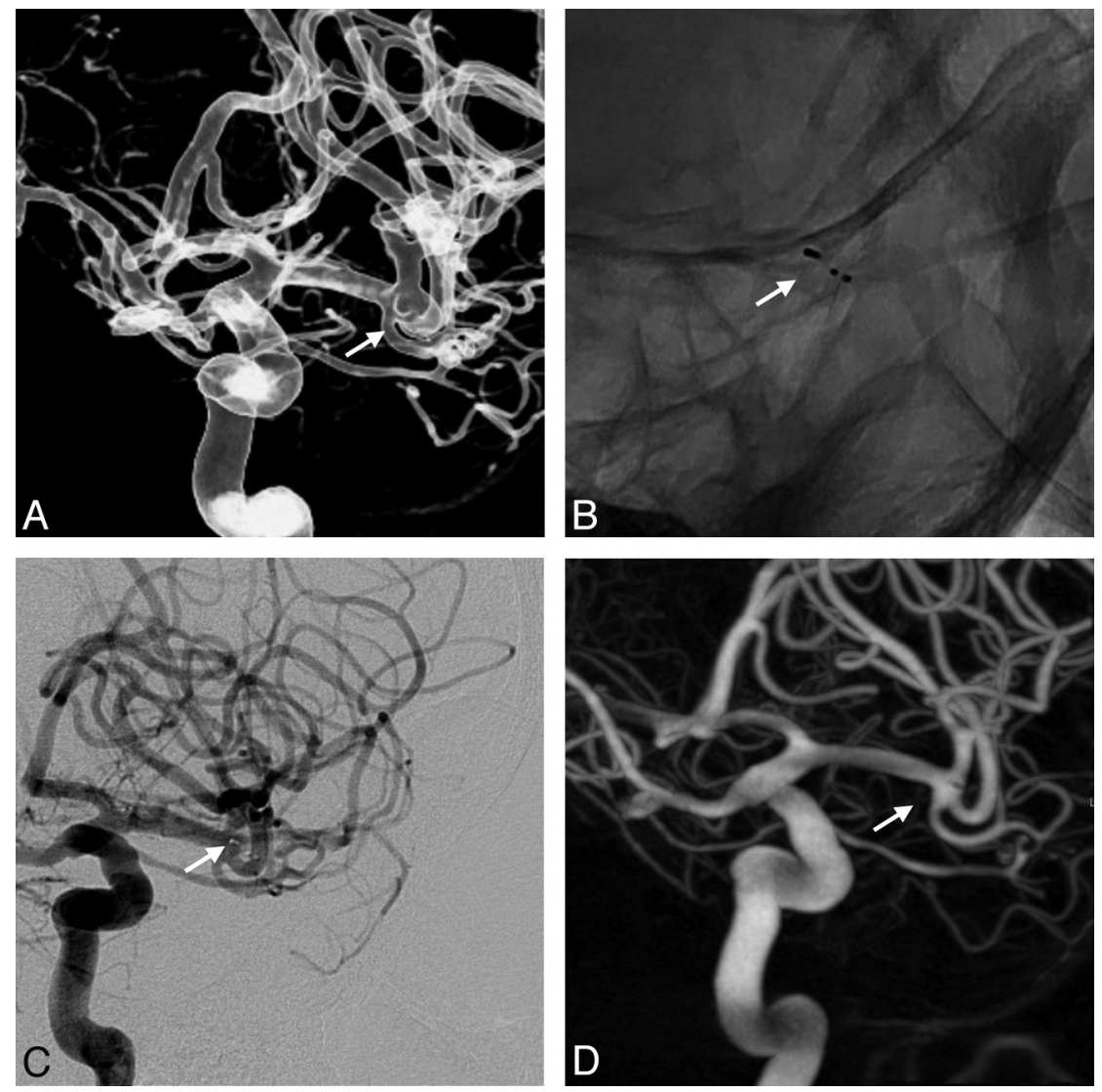

Fig 3. Unruptured middle cerebral artery aneurysm (patient 4). $A, 3 \mathrm{D}$ angiography shows that the aneurysm (arrow) has a wide neck. $B$, Postoperative angiography, oblique unsubstracted view, shows that the WEB is deployed and the 3 markers are visible. $C$, Postoperative angiography, oblique view, shows that the proximal compartment is still filled by the contrast media. $D$, Three-month follow-up angiography shows total occlusion of the aneurysm.

the feasibility of this technique as well as to provide an initial assessment of the safety and efficacy of this treatment.

The WEB is a 2-compartment nitinol device that is inserted into the aneurysm to disrupt the intra-aneurysmal flow and subsequently create intra-aneurysmal (and intradevice) thrombosis (Fig 1). The WEB device must be introduced through a larger microcatheter compared with that used for coiling, and this is a potential limitation of the technique. Preclinical animal studies assessed the feasibility of this approach as well as its safety and efficacy. ${ }^{11}$ The published clinical experience with this device was, until now, limited to a case report. ${ }^{12}$

In this feasibility study, no precise inclusion or exclusion criteria were defined. The idea was to treat mostly aneurysms that were not accessible to regular coiling due to unfavorable anatomy. Unfavorable anatomy is, in fact, not so precisely defined in the literature. Large and giant aneurysms are part of this group. Wide-neck aneurysms are also included in this group and were defined in the present study according to the Humanitarian Device Exemption indications for use of devices approved by the FDA for wide-neck aneurysm treatment (neck width $\geq 4 \mathrm{~mm}$, or dome-to-neck ratio $2: 1$ ). ${ }^{13}$ On the basis of retrospective monocentric series, difficult aneurysms were defined as aneurysms with aspect and dome-to-neck ratios $\leq 1: 6 .{ }^{14}$ According to the FDA definition, 16/21 aneurysms $(76.2 \%)$ had unfavorable anatomies. In 5 cases, the WEB device was used as an alternative to standard coiling because the procedure was expected to be easier or to have a shorter duration.

In this initial selected series, the treatment was successful in all cases $(100.0 \%)$. Treatment by using the device alone was performed in most cases (76.2\%). Additional coiling was planned and used adjunctively to obtain good aneurysm occlusion in 2 giant partially occluded aneurysms. In 2 cases (AcomA aneurysm, MCA aneurysm retreatment) in which the WEB device was undersized, adjunctive coils were used to ensure complete embolization. In the compassionate-use ruptured aneurysm, adjunctive stent placement was successfully performed to prevent rethrombosis of the parent artery. It appears that adjunctive use of coils and stent placement with the WEB device are feasible with an acceptable safety profile.

In this preliminary clinical experience, most aneurysms were bifurcation aneurysms located at the MCA, BA, ICA, and AcomA (19 aneurysms). Certain aneurysm locations (AcomA) proved more challenging to treat. Also, aneurysms with excessive angulation versus the parent artery proved to be more difficult. Recent improvements to the WEB delivery system may facilitate treatment of these aneurysms. As previously outlined, aneurysm anatomy was also an important criterion for WEB indications. Finally, the WEB seems to be well-suited for treatment of wide-neck bifurcation aneurysms or aneurysms located at the MCA, BA, and ICA bifurcation. Other potential indications are AcomA aneurysms and large and 

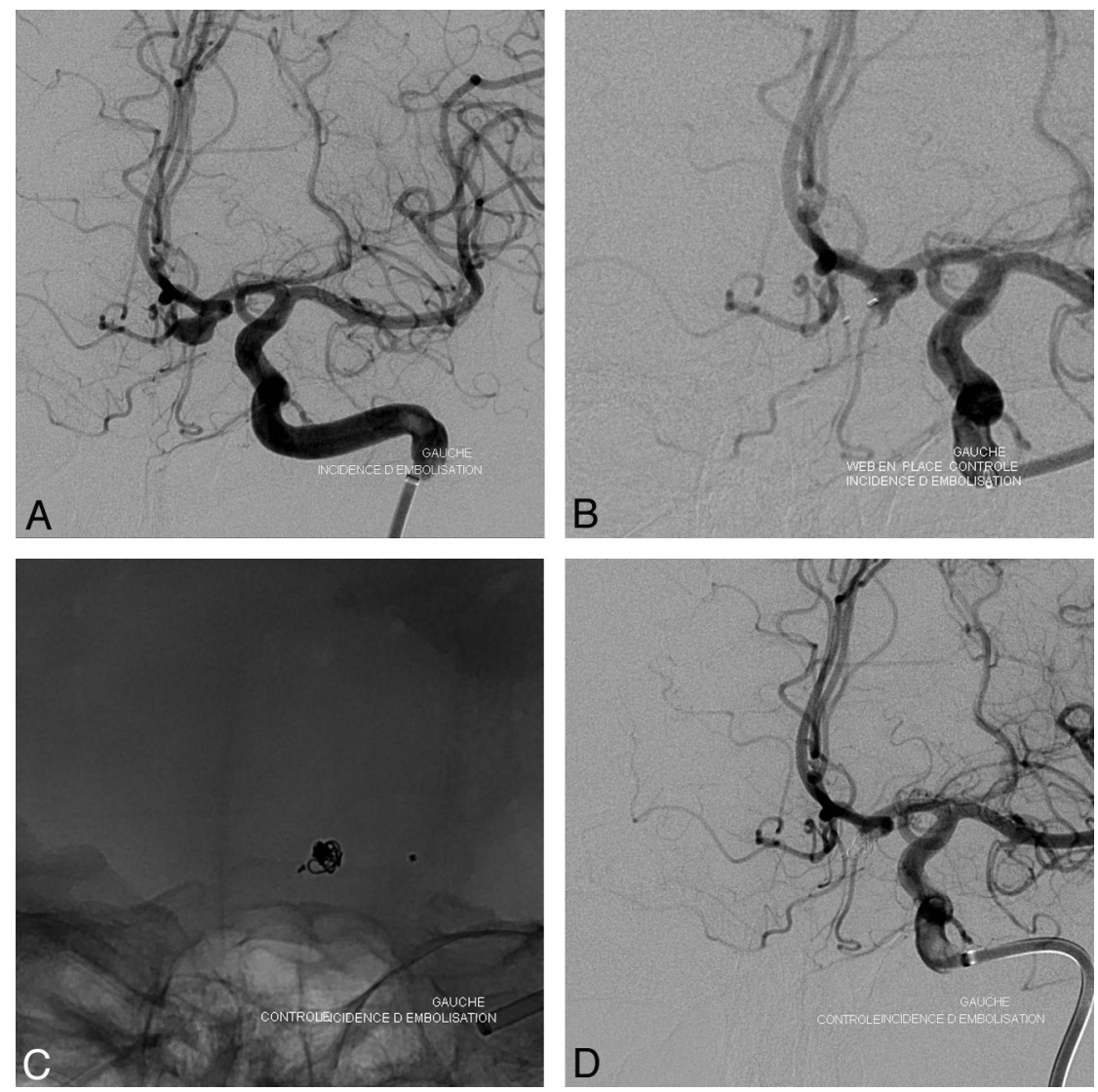

Fig 4. Unruptured anterior communicating artery aneurysm (patient 3). $A$, Preoperative angiography, oblique view, shows the aneurysm. $B$, After detachment of the WEB, a small residual opacification is visible. $C$, Unsubstracted oblique view shows the coils introduced after the WEB treatment. $D$, Final control angiogram shows a complete occlusion of the aneurysm.

giant aneurysms with additional treatment with coiling and/or stent placement.

In the present series, most patients were treated for unruptured aneurysms (except patient 18), mostly due to the logistic organization (devices not present in the departments), and antiplatelet therapy was conducted according to the routine protocol for unruptured aneurysms of the center. However, because no pre- or intraoperative antiplatelet treatment is needed for WEB treatment and because a rapid restriction of flow is observed at the level of the aneurysm dome, it is potentially also applicable to ruptured aneurysms.

In our selected series, the rate of adverse events and morbidity/mortality compares favorably with that of large series of patients treated with coiling. ${ }^{2,3}$ At short-term follow-up (3-12 months), adequate occlusion (total occlusion or neck remnant) was observed in a high percentage of aneurysms (12/15, $80.0 \%)$. An aneurysm remnant was observed in 3 aneurysms $(3 / 15,20.0 \%)$, and retreatment was performed in 2 aneurysms $(2 / 21,9.5 \%)$.

Because the WEB is mostly indicated in wide-neck bifurcation aneurysms, it is an alternative to balloon-remodeling treatment, stent placement, flow diversion, or clipping. The balloon remodeling technique has proved to be safe and efficacious for ruptured and unruptured aneurysms with a sidewall geometry; it is less so and more difficult in bifurcation aneurysms. ${ }^{7}$ In such situations, it is often necessary to protect $>1$ bifurcation branch. A double-balloon technique can be used, but it is technically more challenging. Moreover, the risk of inflating 2 balloons simultaneously in the same usually small vessel has, until now, not been precisely evaluated, to our knowledge. Because patients treated with stent-assisted coiling or flow diversion have to be treated with double antiplatelet therapy, these techniques are typically reserved for patients with unruptured aneurysms. Again these techniques are very helpful for the treatment of sidewall aneurysms, but their use is more limited in bifurcation aneurysms. Y-stent placement or waffle-cone stent placement has been proposed to overcome the limitation of the use of stent placement in bifurcation aneurysms, but the safety of these techniques has yet to be established in a large series of patients and the long-term effect of 2 intermingled stents in a small vessel like the MCA remains unknown, for example. ${ }^{10}$ The use of intraluminal flow diverters for the treatment of bifurcation aneurysms is also not simple. The flow diverter has to be placed in 1 of the bifurcation branches. Depending on the anatomic situation, the neck coverage will not always be optimal and other bifurcation branches will be covered by the flow diverter with the potential risk of occlusion.

Our study had some limitations. First, the number of patients is small, but it was important to have a preliminary evaluation of this very new endovascular approach for aneurysm treatment. Second, midterm and long-term follow-up is needed to evaluate the true efficacy of this treatment in terms of aneurysm recanalization; the recurrence rate relative to coils is largely unknown at this time. 


\section{Conclusions}

Intrasaccular flow disruption by using the WEB is a new endovascular approach for aneurysm treatment. In this preliminary selected series, this treatment was feasible in all cases. The WEB has demonstrated its suitability in treating wideneck bifurcation aneurysms, especially those located on the BA, ICA, and MCA. Further studies should be conducted to evaluate the feasibility, safety, efficacy, and clinical utility of this new treatment.

Disclosures: Laurent Pierot-RELATED: Consulting Fee or Honorarium: Sequent; UNRELATED: Consultancy. Codman, ev3, Penumbra. Thomas Liebig—RELATED: Consulting Fee or Honorarium: proctor for said intrasaccular flow diverter, Sequent, UNRELATED: Consultancy. ev3, Concentric, Comments: Proctoring of Pipeline Embolic Device by ev3, consultant for both ev3 and Concentric/Stryker. Joachim Klisch—RELATED: Consulting Fee or Honorarium: proctoring and consulting for Sequent Medical;* UNRELATED: Consultancy. proctoring for Covidien, ev3. *Money paid to the institution.

\section{References}

1. Molyneux A, Kerr R, Stratton I, et al, for the International Subarachnoid Aneurysm Trial (ISAT) Collaborative Group. International Subarachnoid Aneurysm Trial (ISAT) of neurosurgical clipping versus endovascular coiling in 2143 patients with ruptured intracranial aneurysms: a randomised trial. Lancet 2002;360:1262-63

2. Cognard C, Pierot L, Anxionnat R, et al, for the Clarity Study group. Results of embolization used as the first treatment choice in a consecutive non selected population of ruptured aneurysms: clinical results of the Clarity GDC study. Neurosurgery 2011;69:837-41

3. Pierot L, Spelle L, Vitry F, for the ATENA Investigators. Immediate clinical outcome of patients harboring unruptured intracranial aneurysms treated by endovascular approach: results of the ATENA trial. Stroke 2008;39: 2497-504

4. Ferns SP, Sprengers ME, von Rooij WJ, et al. Coiling of intracranial aneurysms: a systematic review on initial occlusion and reopening and retreatment rates. Stroke 2009; 40:e523-e29

5. Pierot L, Spelle L, Leclerc C, et al. Endovascular treatment of unruptured intracranial aneurysms: comparison of safety of remodeling technique and standard treatment with coils. Radiology 2009;251:846-55

6. Pierot L, Cognard C, Anxionnat R, et al. The remodelling technique for endovascular treatment of ruptured intracranial aneurysms had a higher rate of adequate occlusion than did conventional coil embolization with comparable safety. Radiology 2011;258:546-53

7. Pierot L, Cognard C, Spelle L, et al. Safety and efficacy of balloon remodelling technique during endovascular treatment of intracranial aneurysms: critical review of the literature. AJNR Am J Neuroradiol 2012;33:12-15

8. Piotin M, Blanc R, Spelle L, et al. Stent-assisted coiling of intracranial aneurysm: clinical and angiographic results in 216 consecutive aneurysms. Stroke 2010;41:110-15

9. Pierot L. Flow diverter stents in the treatment of intracranial aneurysms: where are we? J Neuroradiol 2011;38:40-46

10. Lozen A, Manjila S, Rhiew R, et al. Y-stent-assisted coil embolization for the management of unruptured cerebral aneurysms: report of 6 cases. Acta Neurochir (Wien) 2009;151:1663-72

11. Ding YH, Lewis DA, Kadirvel R, et al. The Woven EndoBridge: a new occlusion device. AJNR Am J Neuroradiol 2011;32:607-11

12. Klisch J, Sychra V, Strasilla C, et al. The Woven EndoBridge cerebral aneurysm embolization device (WEB II): initial clinical experience. Neuroradiology 2011;53:599-607

13. Mocco J, Snyder KV, Albuquerque FC, et al. Treatment of intracranial aneurysms with the Enterprise stent: a multicenter registry. J Neurosurg 2009;110: 35-39

14. Brinjikji W, Cloft HJ, Kallmes DF. Difficult aneurysms for endovascular treatment: overwide or undertall? AJNR Am J Neuroradiol 2009;30:1513-17 